Swarthmore College

Works

$5-1-2019$

\title{
Magnetothermodynamics: An Experimental Study Of The Equations Of State Applicable To A Magnetized Plasma
}

M. Kaur

Michael R. Brown

Swarthmore College, doc@swarthmore.edu

A. D. Light

Follow this and additional works at: https://works.swarthmore.edu/fac-physics

Part of the Physics Commons

Let us know how access to these works benefits you

\section{Recommended Citation}

M. Kaur, Michael R. Brown, and A. D. Light. (2019). "Magnetothermodynamics: An Experimental Study Of The Equations Of State Applicable To A Magnetized Plasma". Physics Of Plasmas. Volume 26, Issue 5. DOI: $10.1063 / 1.5083623$

https://works.swarthmore.edu/fac-physics/386

This work is brought to you for free by Swarthmore College Libraries' Works. It has been accepted for inclusion in Physics \& Astronomy Faculty Works by an authorized administrator of Works. For more information, please contact myworks@swarthmore.edu. 


\section{Magnetothermodynamics: An experimental study of the equations of state applicable to a magnetized plasma $\odot$}

Cite as: Phys. Plasmas 26, 052506 (2019); https://doi.org/10.1063/1.5083623

Submitted: 29 November 2018 . Accepted: 18 April 2019. Published Online: 13 May 2019

M. Kaur (D), M. R. Brown (D), and A. D. Light (D)

\section{COLLECTIONS}

Paper published as part of the special topic on Papers from the 60th Annual Meeting of the APS Division of Plasma Physics

Note: This paper is part of the Special Collection: Papers from the 60th Annual Meeting of the APS Division of Plasma Physics. Note: Paper YI3 3, Bull. Am. Phys. Soc. 63 (2018).

F This paper was selected as Featured
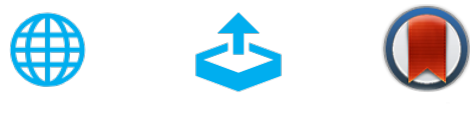

\section{ARTICLES YOU MAY BE INTERESTED IN}

On the generalized formulation of Debye shielding in plasmas

Physics of Plasmas 26, 050701 (2019); https://doi.org/10.1063/1.5091949

\section{Approaching a burning plasma on the NIF}

Physics of Plasmas 26, 052704 (2019); https://doi.org/10.1063/1.5087256

Three-dimensional modeling and hydrodynamic scaling of National Ignition Facility implosions Physics of Plasmas 26, 050601 (2019); https://doi.org/10.1063/1.5091449

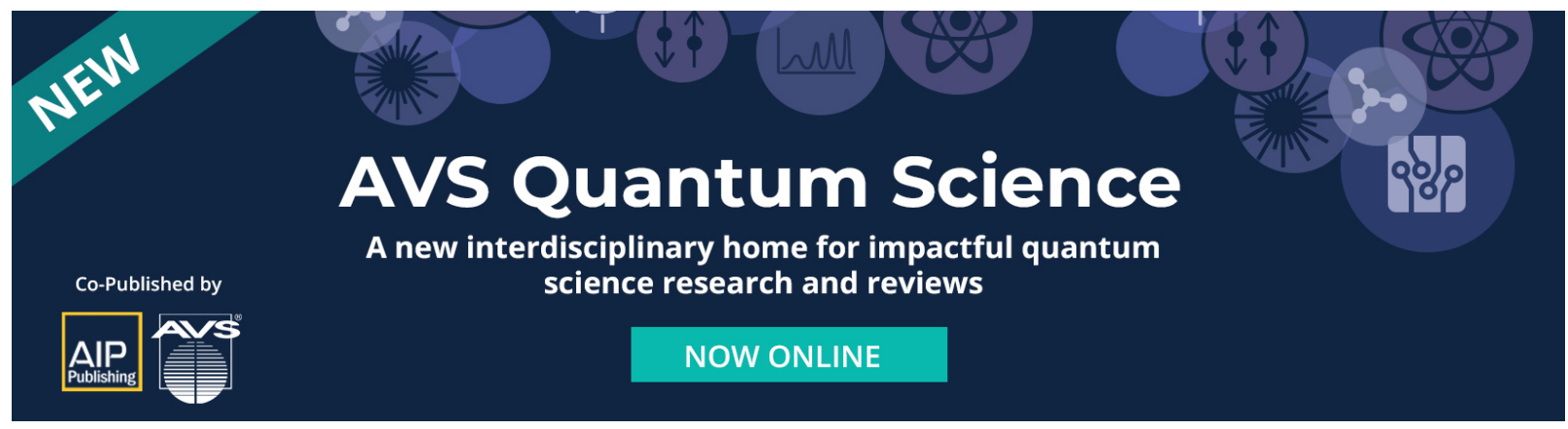




\title{
Magnetothermodynamics: An experimental study of the equations of state applicable to a magnetized plasma
}

Cite as: Phys. Plasmas 26, 052506 (2019); doi: 10.1063/1.5083623

Submitted: 29 November 2018 - Accepted: 18 April 2019 •

Published Online: 13 May 2019

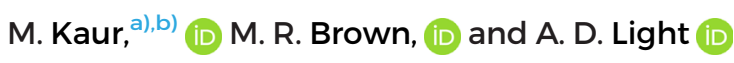

\author{
AFFILIATIONS \\ Department of Physics and Astronomy, Swarthmore College, Swarthmore, Pennsylvania 19081, USA \\ Note: This paper is part of the Special Collection: Papers from the 60th Annual Meeting of the APS Division of Plasma Physics. \\ Note: Paper YI3 3, Bull. Am. Phys. Soc. 63 (2018). \\ a) Invited speaker. \\ b) Electronic mail: mkaur2@swarthmore.edu
}

\begin{abstract}
Measuring the equations of state of a compressed magnetized plasma is important for both advancing fusion experiments and understanding natural systems such as stellar winds. In this paper, we present results from our experiments on the thermodynamics of compressed magnetized plasmas; we call these studies "magnetothermodynamics." In these experiments, we generate parcels of relaxed, magnetized plasma at one end of the linear Swarthmore Spheromak eXperimental device and observe their compression in a closed conducting boundary installed at the other end. Plasma parameters are measured during compression. Instances of ion heating during compression are identified by constructing a pressure-volume diagram using the measured density, temperature, and volume of the magnetized plasma. An axial scan of the ion temperature at upstream locations suggests that the increase in ion temperature arises due to the compression of the magnetized plasma in the conducting boundary. The theoretically predicted magnetohydrodynamic (MHD) and double adiabatic equations of state are compared with experimental measurements to estimate the adiabatic nature of the compressed plasma. The equilibrium of our magnetized plasmas is well-described by magnetohydrodynamics; however, we find that the MHD equation of state is not supported by our data. Our results are more consistent with the parallel Chew-Goldberger-Low equation of state, suggesting that there is significant anisotropy in the ion distribution function.
\end{abstract}

Published under license by AIP Publishing. https://doi.org/10.1063/1.5083623

\section{INTRODUCTION}

An experimental study of the determination of the equations of state (EOS) applicable to a compressible magnetized plasma is essential for realizing controlled nuclear fusion. Numerous laboratory experiments have been performed to achieve a highly compressed plasma to ultimately lead toward fusion such as inertial confinement fusion experiments on unmagnetized plasma, ${ }^{1,2}$ experiments on magnetically confined plasmas, ${ }^{3-7}$ and magnetized liner inertial fusion experiments. ${ }^{8-10}$ However, very little has been done to understand the thermodynamics of these magnetized plasmas.

We have been exploring the thermodynamics of compressed magnetized plasmas (referred to as magnetothermodynamics) using measured plasma parameters in a stagnating Taylor state, as described in the study by Kaur et al. ${ }^{11,12}$ The main motivation behind these thermodynamics studies is to explore the possibility of producing dense hot plasma by compressing them using their own inertia. In this paper, we review these results and present our latest findings. The Swarthmore Spheromak eXperimental (SSX) device launches a plasma which is compressed by stagnation in a closed conducting volume. To identify events corresponding to adiabatic heating, we use the measured plasma parameters, such as density, temperature, and magnetic field, to construct pressure-volume (PV) diagrams. Axial scans of the ion temperature confirm that the ion heating occurs due to compression of the magnetized plasma against a conducting wall. The heating events are used to identify the dominant equation of state applicable to our magnetized plasma. In these experiments, we found that the dynamics of SSX plasmas is determined by the parallel ChewGoldberger-Low (CGL) equations of state.

Figure 1 shows analytically solved streamlines of the magnetic field for a Taylor state $e^{13,14}$ by solving the linear eigenvalue problem 


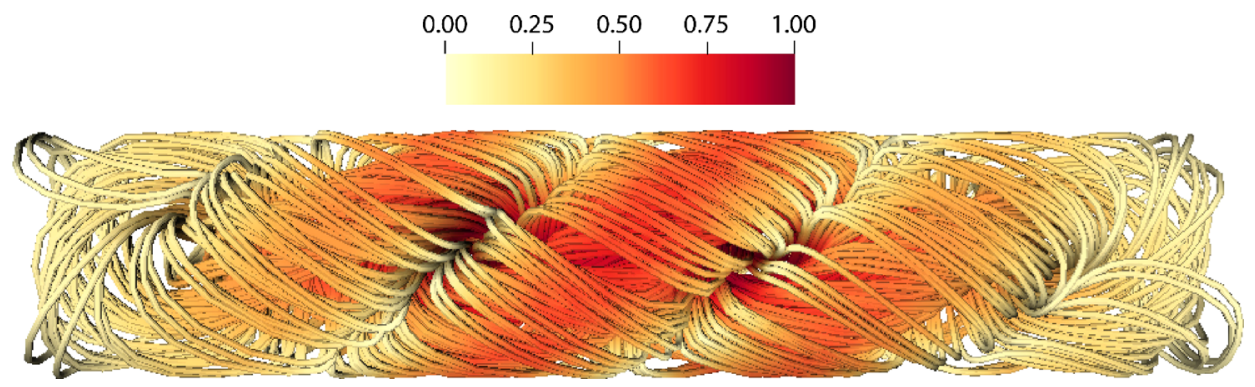

FIG. 1. A 3D arrangement of the magnetic field lines in a twisted Taylor state plotted using Vislt. The relative magnitude of $|B|$ at any location is plotted using the colorbar at the top.

$\nabla \times \mathbf{B}=\lambda \mathbf{B}$ with the PSI-center TETrahedral mesh (PSI-TET) code $^{33}$ for a 10:1 aspect ratio (ratio of the length of a conducting boundary to its radius) and plotted using VisIt. ${ }^{15}$ The color represents the strength of the magnetic field, normalized to its maximum value. The ivory color corresponds to a weak magnetic field, whereas the dark red color indicates a strong magnetic field. The conducting cylindrical boundary with an aspect ratio $>10$ acts as a quasi-infinite cylinder, and the minimum energy state for such a boundary corresponds to the $\lambda a=3.11$ solution. ${ }^{14}$ This nonaxisymmetric double helix state is found to have a helical pitch of $k a=1.29$, where $k$ is the wave number associated with the $\mathrm{z}$ axis and $a$ is the radius of the cylinder. In our experiments, we measure the value of $k$ from the magnetic field data as a proxy for the length of the Taylor state.

One important feature of these Taylor state plasmas is that one does not need to apply any external magnetic field for their confinement. In this way, these plasmas are different from tokamak plasmas where a careful configuration of external magnetic fields is required to sustain the plasma. The Taylor state is a self-contained object where the magnetic field is generated due to the flow of current in the plasma and the current carrying charged particles are confined by the selfgenerated magnetic field. Therefore, it serves as an ideal closed system for performing this type of thermodynamic studies.

The remainder of this paper is organized as follows. In Sec. II, we briefly review the different equations of state applicable to a magnetized plasma. In Sec. III, we discuss the experimental setup used to perform these studies and our main diagnostics used to carry out different measurements. In Sec. IV, we show results related to ion heating and the tests on various equations of state followed by a brief discussion. In Sec. V, we summarize the results and discuss our future plans.

\section{THEORY}

We consider three equations of state (EOS) for our magnetized plasmas. We ignore the radiation effects. In the magnetohydrodynamic (MHD) regime, plasma with isotropic velocity distribution can be treated as an ideal gas and the adiabatic equation of state for such a plasma is given by

$$
\frac{\partial}{\partial t}\left(\frac{P}{n^{\gamma}}\right)=0
$$

where $P$ and $n$ are the plasma pressure $\left(P=n k_{B} T\right)$ and density, respectively; $T$ is the plasma temperature and for an MHD plasma with three degrees of freedom, $\gamma=5 / 3$.

If the collisionality is low, i.e., the ion-ion collision frequency is less than the ion cyclotron frequency, it is hard to maintain an isotropic velocity distribution. In such a case, the perpendicular and parallel pressures to the magnetic field are not same and the MHD equation of state no longer remains valid. To account for such a situation, Chew et al. ${ }^{16}$ proposed a modified adiabatic equation of state model, known as the CGL equations of state

$$
\begin{aligned}
\frac{\partial}{\partial t}\left(\frac{P_{\perp}}{n B}\right) & =0, \\
\frac{\partial}{\partial t}\left(\frac{P_{\|} B^{2}}{n^{3}}\right) & =0 .
\end{aligned}
$$

Equation (2) is related to the constancy of the first adiabatic invariant, $\mu=W_{\perp} / B$, and Eq. (3) is related to the constancy of the second adiabatic invariant, $\mathcal{J}=v_{\|} L$, where $W_{\perp}, v_{\|}$, and $L$ are the perpendicular kinetic energy with respect to the magnetic field, parallel velocity with respect to the magnetic field, and the length of the guiding center motion along the field lines, respectively.

We test our data from SSX plasmas against these three equations of state (EOS). If the time derivative of any of these measured quantities is zero during a heating event, that particular equation of state would be valid for our plasma. Since the equilibrium of the twisted Taylor state is determined by magnetohydrodynamics, it is reasonable to expect that it can be described by the MHD equation of state. On the other hand, because of the strong magnetic field embedded in the structure and the low collisionality, confined particles could support different parallel and perpendicular velocity distributions. In that case, the Taylor states may follow the CGL double adiabatic equations of state.

\section{EXPERIMENTAL SETUP AND DIAGNOSTICS}

We produce parcels of magnetized plasma using a coaxial magnetized plasma gun, which is located at one end of the linear Swarthmore Spheromak eXperimental (SSX) device, as shown in Fig. 2. Details about the plasma gun can be found in earlier publications. ${ }^{17-19}$ At the other end of the linear chamber, $\mathrm{a} \sim 30 \mathrm{~cm}$ long closed, tungsten-lined copper can, referred to here as a stagnation flux conserver (SFC), is installed. A $1 \mathrm{~m}$ long glass tube (inner diameter $=15 \mathrm{~cm}$ ) is installed in between the gun and the SFC. To provide flux conservation to the magnetized plasma, the glass tube has been covered with a copper flux conserving shell with a 10:1 aspect ratio and a long magnetic soak time. The magnetic soak time is the time magnetic lines of force would take to diffuse through the conductor and is given by $\tau_{m}=\mu_{0} r \delta / 2 \rho$, where $\delta, r$, and $\rho$ are the thickness of the conductor used for preventing the magnetic field diffusion, radius of the conducting shell, and its electrical resistivity, respectively. For the copper flux 


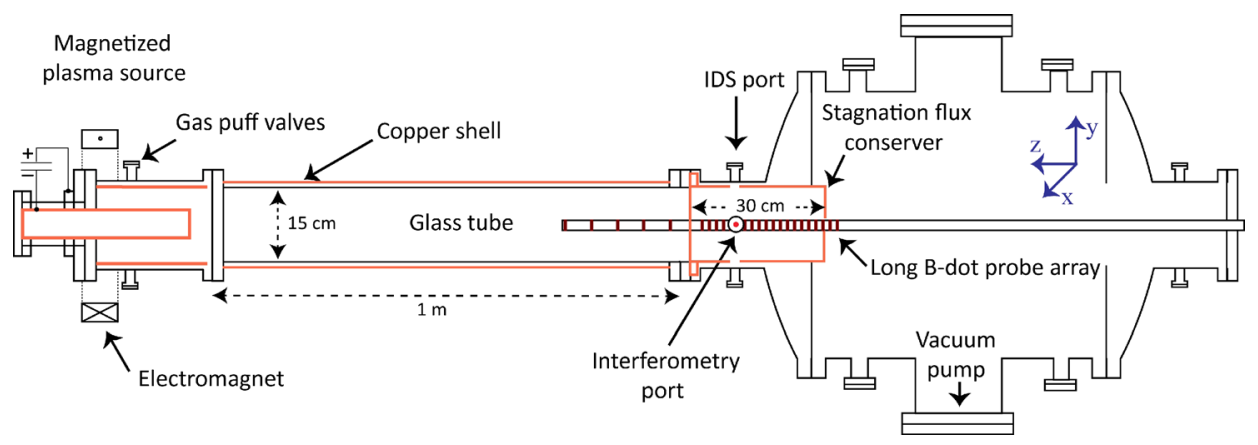

FIG. 2. A schematic of the experimental setup along with the diagnostic ports. The plasma source is located at the left end of the linear SSX device and at the other end, a closed conducting boundary (referred to as the stagnation flux conserver) is installed, indicated by the red color. A glass tube is mounted in between the gun and the closed boundary and is covered with a copper flux conserving shell (also indicated by the red color). Principal plasma diagnostics are located in the SFC to measure the plasma compression. The ion Doppler spectroscopy telescope is mounted vertically, along the $y$-axis, to measure $T_{i}$, and $n$ is measured using HeNe laser interferometry along the $x$-axis. The long $\dot{B}$ probe array is aligned along the axis of the SFC (along the $z$-axis). The $\dot{B}$ probes (represented by the maroon lines) are densely spaced in the SFC to measure the magnetic field structure and time of flight velocity of the plasma.

conserver being utilized, the magnetic soak time is greater than $200 \mu \mathrm{s}$ and our plasma lifetime is $\sim 100 \mu \mathrm{s}$.

Initially, the gun is prepared with a strong magnetic field $(\sim 1 \mathrm{~T})$ in the inner electrode using an external electromagnet (stuffing field). A hydrogen gas puff is applied into the annular region between the two electrodes using gas puff valves. A voltage pulse $(\approx 4 \mathrm{kV}, 8 \mathrm{~kJ})$ is applied between the two electrodes (at $0 \mu \mathrm{s}$ ), which ionizes the gas present in the annular region and a high current $(\sim 100 \mathrm{kA})$ flows through the plasma. The current causes a strong magnetic field that gives rise to a $\mathbf{J} \times \mathbf{B}$ force which accelerates the plasma out of the gun, like a rail gun, whereas the stuffing field initially keeps the plasma confined to the annular region. If the $\mathbf{J} \times \mathbf{B}$ force is strong enough, the stuffing field bulges outward around the accelerating plasma and eventually a self-consistent toroidal magnetic structure, called a spheromak, ${ }^{18}$ is formed at around $20-25 \mu$ s.

After formation, the spheromak travels at $v \approx 40 \mathrm{~km} / \mathrm{s}$, consistent with the $\mathbf{J} \times \mathbf{B}$ force, away from the gun due to its inertia. The spheromak is not the lowest energy equilibrium for the long aspect ratio flux conserver. Therefore, it tilts and relaxes to a nonaxisymmetric twisted, force-free, minimum energy state, called a Taylor state. ${ }^{14,19,20}$ The relaxation process is highly turbulent in nature and happens within a couple of Alfvén times. The center-of-mass inertia carries the relaxing Taylor state to the other end of the device (into the SFC), where it stagnates and compresses against the end wall. Compression is mostly expected to happen near the back end of the SFC. We perform measurements $20 \mathrm{~cm}$ upstream in the absence of diagnostic ports near the back end of the SFC.

We rely on three principal diagnostics located in the SFC. To measure submicrosecond time-resolved ion temperature with an instrument temperature of 3-5 eV, we make use of ion Doppler spectroscopy (IDS). Our IDS system features a $1.33 \mathrm{~m}$ (Czerny-Turner) spectrometer which makes use of an echelle grating (with 316 groove/ $\mathrm{mm}$ ) to achieve a high spectral resolution and a 32-channel photomultiplier tube (PMT) array for fast time response. ${ }^{21}$ The plasma light is collected along a vertical chord, dispersed on the echelle grating, and is recorded using the PMT array. Our current studies are focused on the evolution of $229.687 \mathrm{~nm}$ emission lines from $\mathrm{C}_{I I I}$ impurity ions present in our plasma, ${ }^{22}$ which we observe at the 25 th order.
For measuring the plasma density, we use a HeNe laser interferometer. It is a Mach-Zehnder arrangement where laser beam is split into two beams, one passes through the plasma and the refractive index of the plasma introduces a phase shift. The interference signal is passed through a Wollaston prism which converts it into two outputs $90^{\circ}$ out of phase with each other. This arrangement enables us to measure the plasma density during each shot.

In order to measure the helical pitch of the Taylor state, we use a long $\dot{B}$ probe array installed along the axis of the SFC. We use 14 bit D-Tacq digitizers at a high cadence $(65 \mathrm{MHz})$ to record the $\dot{B}$ probe data, which is later numerically integrated using the trapezoidal rule to obtain the absolute magnetic field values. Since the magnetic probe array is aligned along the axis of the SFC, we see a helical structure of the relaxed Taylor state as it enters the SFC.

The typical SSX plasma parameters in the SFC are as follows. The plasma density $(n)$ is $\sim 2 \times 10^{21} \mathrm{~m}^{-3}$. The electron temperature $\left(T_{e}\right)$ measured using vacuum ultraviolet (VUV) spectroscopy is $\sim 10 \mathrm{eV}$ and remains nearly constant. ${ }^{23}$ The ion temperature $\left(T_{i}\right)$ varies from 10 to $40 \mathrm{eV}$ and the magnetic field $(B)$ embedded in the Taylor state is $\sim 2000 \mathrm{G}$. For these plasma parameters, the ion gyroradius and the Alfvén speed are $\sim 2 \mathrm{~mm}$ and $\sim 100 \mathrm{~km} / \mathrm{s}$, respectively and the plasma $\beta$ varies from 0.1 to 1 .

\section{RESULTS AND DISCUSSION}

In this section, we discuss the results obtained using different diagnostics and the EOS analysis on a compressed plasma.

The plasma moves at $40 \mathrm{~km} / \mathrm{s}$ and reaches the SFC at $\sim 40 \mu \mathrm{s}$. Figure 3 shows a vector rendering of the magnetic field along the axis of the SFC obtained from the long $\dot{B}$ probe array (Multimedia view). The object enters from the right-hand side and the solid black lines indicate the end of the SFC. As the object moves inside the SFC, we see a right-handed helical magnetic field structure. The helical pitch confirms that by the time the plasma reaches the SFC, it has already relaxed to a twisted Taylor state. Using this magnetics data, we extract the information about the time varying pitch of the Taylor state which serves as a proxy for the length of the Taylor state. We use the fractional time variation in length to determine the fractional time variation in volume. 

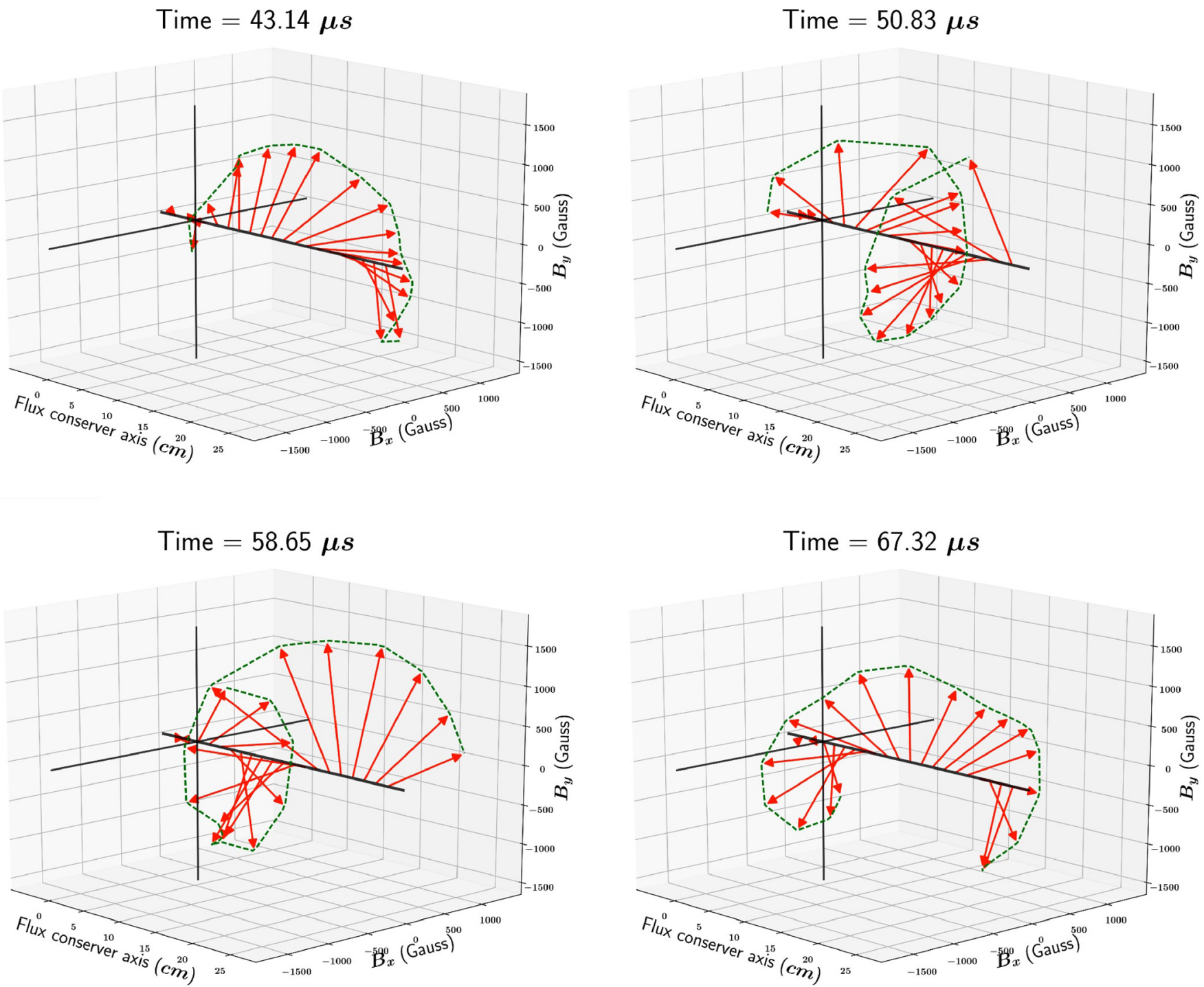

FIG. 3. A four-panel plot showing the vector rendering of the Taylor state inside the SFC at different times. The plasma enters the SFC at $\sim 40 \mu$ sand fills the entire SFC at $43 \mu \mathrm{s}$. At later times, we see the plasma being compressed and more than one lobe entering the SFC. Multimedia view: https://doi.org/10.1063/1.5083623.1

Figure 4 shows a typical time trace of the (a) density, (b) ion temperature, and (c) magnetic field from a three-directional $\dot{B}$ probe colocated with the interferometry and IDS chords. As the plasma stagnates and gets compressed against the end wall, we observe ion heating from $65 \mu$ s to $67 \mu$ s (indicated by the red color). Since the plasma flow velocity is much less than the ion sound speed $(\sim 70 \mathrm{~km} / \mathrm{s})$ and Alfvén speed $(\sim 130 \mathrm{~km} / \mathrm{s})$, plasma stagnation, and compression (due to its own inertia) against the SFC end wall is not expected to produce shocks.

To confirm that the ion heating is arising due to the compression of the plasma in the SFC, we carried out an axial scan of the ion temperature (at four axial locations away from the gun), as shown in Fig. 5 . We record a number of shots, around 20-30, at each axial location and calculate the averages. From the axial scan of $\left\langle T_{i}\right\rangle$ at each axial location, we found that the gun generates a hot plasma (shown by the $\left\langle T_{i}\right\rangle$ trace at $24 \mathrm{~cm}$ ), which cools down as it moves away from the gun, evident from the $\left\langle T_{i}\right\rangle$ trace at $72 \mathrm{~cm}$ away from the gun. The plasma ion temperature increases again as it reaches the stagnation flux conserver, shown by the $\left\langle T_{i}\right\rangle$ traces at a distance of $92.6 \mathrm{~cm}$ and $124 \mathrm{~cm}$ from the gun. The increase in ion temperature can arise due to a number of factors such as due to turbulence followed by magnetic reconnection or kinetic effects or simply due to compression of plasma.

To check if the ion heating is originating due to any turbulence driven effects, we perform a frequency power spectrum on the magnetic field time traces. A typical power spectrum is shown in Fig. 6(a). The frequency power spectrum shows a steep spectral index $(\approx-11 / 3)$, 

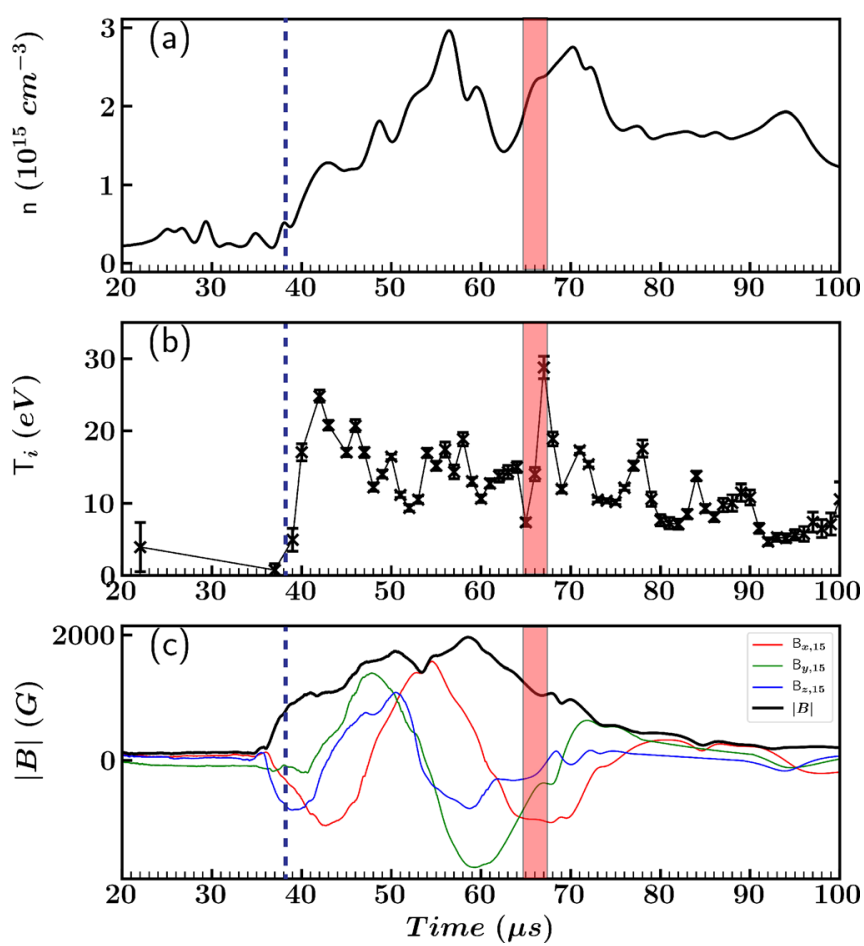

FIG. 4. A typical time trace of (a) the plasma density, (b) ion temperature, and (c) magnetic field measured in the SFC. The leading edge of the Taylor state enters the SFC at $40 \mu \mathrm{s}$ (indicated by the blue dotted line) and we observe a substantial ion heating event at $65 \mu$ s (indicated by the red color).

much steeper than the one observed during the turbulence phase upstream $^{24,25}$ and the usual Kolmogorov scaling ${ }^{26}$ of $-5 / 3$. This indicates that by the time the Taylor state reaches the SFC, there is no turbulence present in the plasma. The steeper slope further confirms that

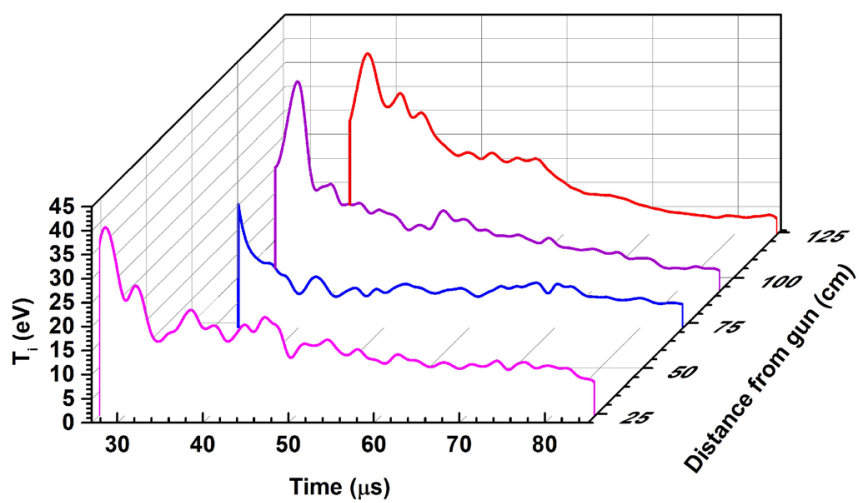

FIG. 5. $T_{i}$ is measured using ion Doppler spectroscopy at four different locations away from the gun averaged over a number of shots at each location. The plasma reaches the end of the SFC at $t \sim 40 \mu \mathrm{s}$. The gun generates a hot plasma, evident from the $\left\langle T_{i}\right\rangle$ trace $24 \mathrm{~cm}$ away from the gun. The plasma cools down as it moves away from the gun, indicated by the $\left\langle T_{i}\right\rangle$ trace $72 \mathrm{~cm}$ away from the gun. The ion temperature increases as the plasma reaches the stagnation flux conserver, shown by the $\left\langle T_{i}\right\rangle$ traces $92.6 \mathrm{~cm}$ and $124 \mathrm{~cm}$ away from the gun. the ion heating is arising solely due to the stagnation and compression of the magnetized plasma against the conducting SFC wall.

In addition, we calculate the probability distribution function (PDF) of increments ${ }^{27}$ constructed by taking the differences of $\dot{B}_{x}\left(\dot{B}_{y}\right.$ or $\dot{B}_{z}$ ) time series separated by a time scale $\Delta t$, i.e.,

$$
\Delta \dot{B}_{x}=\dot{B}_{x}(t+\Delta t)-\dot{B}_{x}(t) .
$$

The PDF of increments for $\Delta t=1 \mu \mathrm{s}$, plotted in Fig. 6(b), is close to a Gaussian distribution confirming no turbulence. We also observe that the magnetic field fluctuations (root mean square) are almost absent during compressive heating events confirming that the compression of the magnetized plasma or a rise in thermal pressure does not drive fluctuations in the plasma.

The time traces of plasma density and ion temperature are used to calculate the plasma thermal pressure for constructing a PV
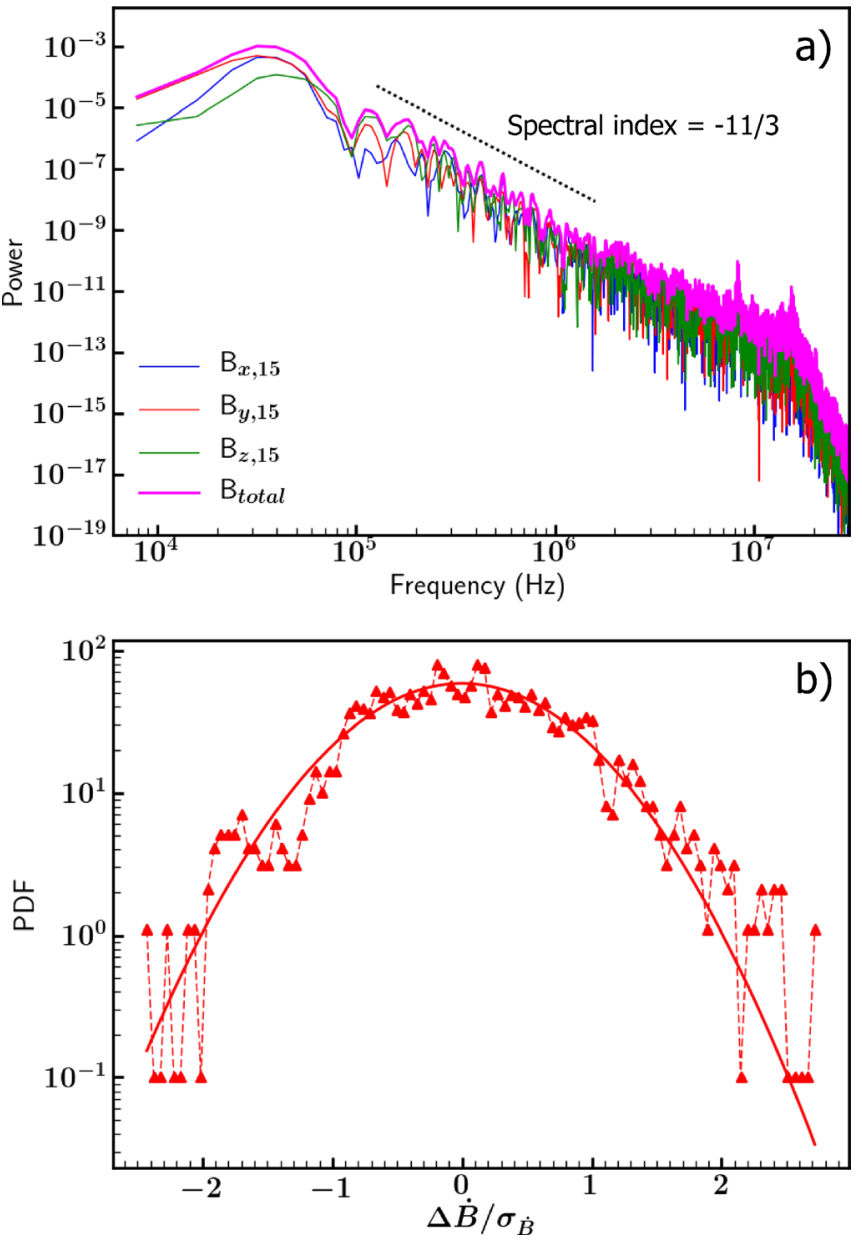

FIG. 6. (a) A typical frequency power spectrum performed on the magnetic field time traces obtained using the $\dot{B}$ probes located in the SFC and (b) the probability distribution function (PDF) of the increments for $\Delta t=1 \mu \mathrm{s}$. The $\mathrm{x}$-axis is normalized by the standard deviation in $\dot{B}\left(\sigma_{\dot{B}}\right)$. A spectral index steeper than $-11 / 3$ and a Gaussian distribution of increments confirm that no turbulence is active in the plasma by the time it reaches the SFC. 
diagram. Since the electron temperature remains nearly constant and for EOS analysis, we are only interested in time varying quantities, $T_{e}$ is not used for these studies. For approximating the volume of the Taylor state, we assume that the plasma initially fills the entire SFC volume. Fractional changes in the pitch are mapped to fractional changes in the length of the confined plasma and hence to fractional changes in the confined plasma volume.

Figure 7 shows a typical PV diagram from the time traces presented in Fig. 4 corresponding to a big ion heating event at 65-67 $\mu$ s. The ion temperature increases by a factor of 3 for this time and density increases by $25 \%$ [shown in Figs. 4(b) and 4(a), respectively]. From the magnetics data, we find that the length of the Taylor state compresses by $30 \%$ at the same time, as shown in Fig. 7(a). We call this type of event, where the Taylor state compresses, ions are heated, and the PV diagram shows a transition from a lower to a higher isotherm, a compressive heating event.

To see the statistical behavior of the plasma, we recorded a number of shots under the same gun conditions and identified many such compressive heating events. Since the gun generates a slightly different plasma in each shot, the criteria for any event to be called a compressive heating event is as follows:

- Length compression of the Taylor state is equal or greater than $10 \%$, we usually observe a length compression of $10 \%-40 \%$.

- The duration of the event is longer than $1 \mu \mathrm{s}$, which is the time resolution of our IDS system.

- The PV diagram demonstrates a transition from a lower to a higher isotherm.

Using the above criteria, we identified $\sim 120$ compressive heating events. A histogram showing the volume compression (or
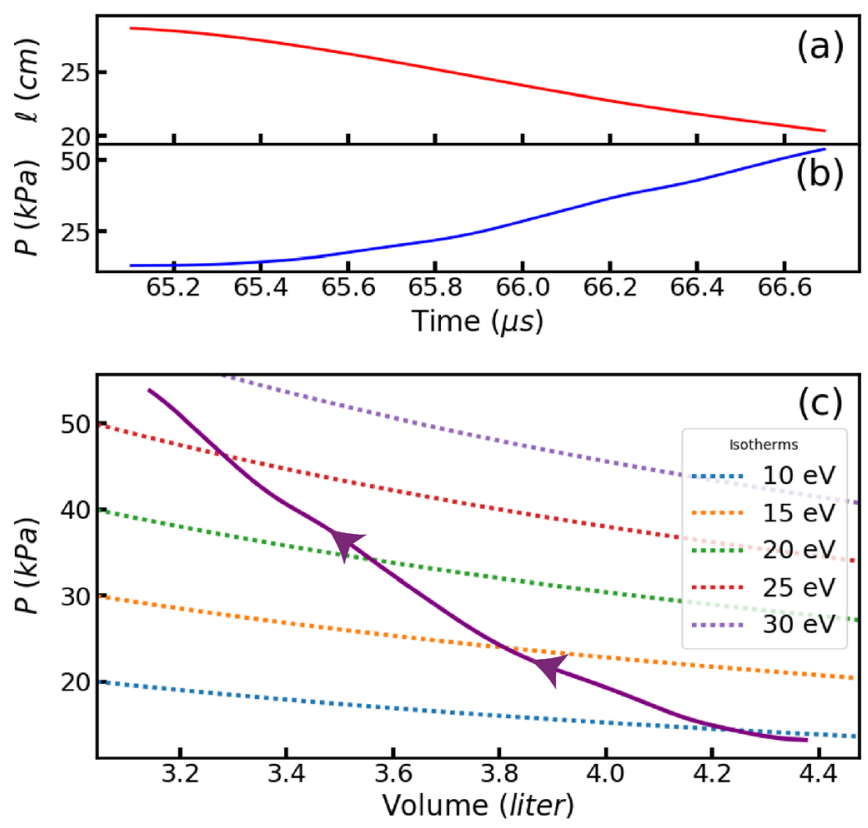

FIG. 7. Typical time trace of the Taylor state length (showing $30 \%$ compression) is shown in (a). Second panel (b) shows an increase in the thermal pressure of the plasma for the same time. The third panel (c) shows a graph of pressure vs volume with different isotherms plotted using the dotted lines in the background.

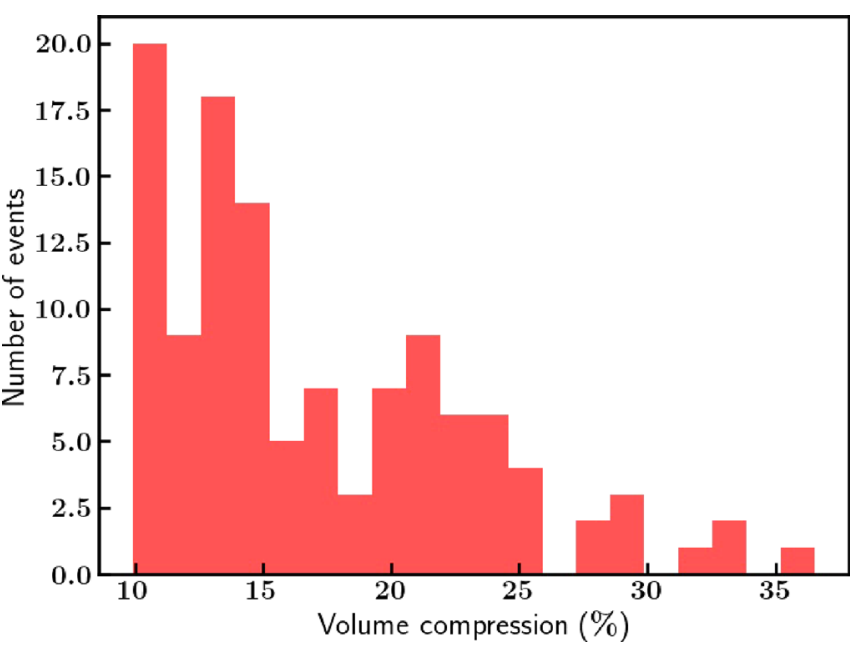

FIG. 8. A histogram showing the compression in the volume of the Taylor state (proportional to the length).

length compression) of the Taylor state is shown in Fig. 8. We used these to analyze the different equations of state shown in Fig. 9. The first panel corresponds to the MHD EOS, and the second and third panels are for the perpendicular and parallel CGL EOS, respectively. The light red lines in each panel show the spread of 120 compressive heating events and the dark red band corresponds to the standard error in the mean of the spread. The black line corresponds to the zero axis. It can be clearly seen that the first two EOS show a nonzero time derivative, and hence are not satisfied during compression. The parallel CGL EOS stays near the zero axis, which indicates that our plasma obeys the parallel CGL equation. The MHD EOS does appear to hold for our plasma, despite the equilibrium being well-described by pure MHD.

These results can be explained in the following way: After formation, the spheromak tilts and relaxes to a twisted Taylor state by minimizing its magnetic energy. During relaxation, the magnetic field embedded in the structure drops by a factor of 10 under the constraint of helicity conservation. Our object lies in the weakly collisional regime so we expect the particle magnetic moment to be an adiabatic invariant. Therefore, if there is a drop in the magnetic field then there should be a corresponding drop in the perpendicular proton energy. We have observed (from the magnetic field vector renderings shown in Fig. 3) that by the time the plasma arrive in the SFC, it has already relaxed to a twisted Taylor state indicating that $T_{\perp}$ has dropped and the proton energy is primarily in $T_{\|}$.

As we mentioned earlier, we measure the ion temperature along a chord and do not distinguish between parallel and perpendicular temperatures. To test the different EOS, we use the total pressure $(P)$ in place of parallel $\left(P_{\|}\right)$and perpendicular $\left(P_{\perp}\right)$ pressures. If protons enter the SFC with most of their energy in the parallel direction, then the total pressure mostly consists of the parallel pressure (i.e., $\left.P=P_{\|}+P_{\perp} \approx P_{\|}\right)$. Thus, even if both CGL equations are satisfied during our compression events, we may not be sensitive to the perpendicular dynamics. In this case, we expect to find consistency with the parallel CGL equation and inconsistency with the perpendicular CGL 


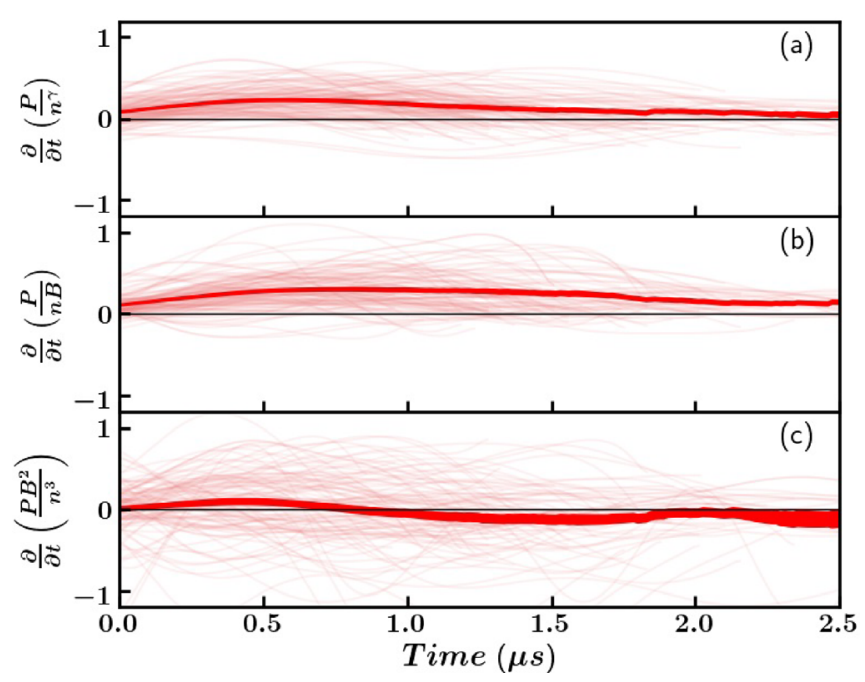

FIG. 9. Statistical variation of the time derivatives of the three equations of state for 120 compression events are shown by the light red lines for: (a) the MHD EOS for 3D compression $(\gamma=5 / 3)$, (b) perpendicular, and (c) parallel CGL EOS. The dark red band in each panel shows the standard error of the mean. The MHD equation of state (a) and the perpendicular CGL EOS (b) have the nonzero time derivative, whereas, the parallel CGL EOS (c) has a nearly zero time derivative for most of the compression time. Adapted with permission from Kaur et al. Phys. Rev. E 97, 011202 (2018). Copyright 2017 The American Physical Society.

equation because the quantity we use as a proxy for $P_{\|}$and $P_{\perp}$ would in fact be primarily $P_{\|}$in both cases.

\section{SUMMARY AND FUTURE PLANS}

A magnetized plasma is launched at $40 \mathrm{~km} / \mathrm{s}$ into a long-aspectratio cylinder by a magnetized coaxial plasma gun. The plasma relaxes to a Taylor state and reaches the other end of the device where it stagnates in a closed conducting boundary and compresses by $\sim 30 \%$. We measure the parameters of the compressed plasma and identify the events corresponding to adiabatic compression of the plasma by constructing PV diagrams. These events are used to statistically identify the equation of state being followed by the compressed plasma. In these experiments, we found that the dynamics of the SSX plasmas is determined by the parallel CGL EOS. This further suggests that the SSX plasma possesses an anisotropic velocity distribution and has more proton energy in the parallel direction with respect to the background magnetic field.

From the axial scan of the ion temperature, we observed that the ion temperature increases as the plasma enters the SFC and gets compressed there. A frequency power spectrum on the magnetics data (in the SFC) showed a steep spectral index $(\sim-11 / 3)$ confirming that there are no turbulence related mechanisms active in the plasma and hence, the ion heating arises solely due to the plasma stagnation and compression.

To aid the existing results, we are planning to carry out the following improvements in our future experiments: Currently, we do not have the flexibility to measure parallel and perpendicular components of ion temperature separately as we perform the ion temperature measurements along a chord. For performing a precise CGL EOS analysis, we are planning to use an ion Doppler spectroscopy probe or an ion energy analyzer (IEA) to enable measurements of the parallel and perpendicular ion temperatures.

Ion Doppler spectroscopy has been utilized in the past on Madison Symmetric Torus (MST) for measuring localized, directional ion temperature. ${ }^{28}$ In this technique, plasma light was collected by two fused silica fiber optic bundles with perpendicular collection chords. To obtain a spatial resolution of $5 \mathrm{~cm}$, the light collection chord was terminated by using an optical dump. A similar technique could be employed in the SSX device. For measuring the direction of the magnetic field, a three-directional $\dot{B}$ probe could be housed in the optical dump which could be placed $\sim 5 \mathrm{~mm}$ away from the IDS fiber.

In such a case, an IDS probe would provide information about the ion temperature over a small chord length. The local plasma density could be measured using a separate colocated probe (maybe a double Langmuir probe operating in the ion saturation region). For minimizing the plasma perturbations and for localized $T_{i}$ measurements, the IDS probe needs to be very compact. This could be done by shortening the length of the light collection chord, which could further result in reduction in the signal. The signal could be increased by replacing $\mathrm{H}_{2}$ with Helium as the working gas and by utilizing the $\mathrm{He}$ line (instead of the $\mathrm{C}_{I I I}$ line) for IDS measurements. A similar technique was employed by Hsu et al. ${ }^{29}$

Another method to measure the directional ion temperature could be to use a planar ion energy analyzer ${ }^{30}$ (IEA), where the ions would be filtered through a microchannel plate prior to collection. ${ }^{31}$ The energy analyzer can be equipped with a three-directional $\dot{B}$ probe to measure the direction of the magnetic field locally. Since the magnetic field embedded in the plasma is much stronger than the magnetic field generated by the ion collection current (from the IEA), it is not expected to affect the $\dot{B}$ probe measurements. However, the dimensions of the microchannels and the interelectrode separation in an IEA should be smaller than the plasma Debye length $(\sim 1 \mu \mathrm{m}$ for SSX plasmas) and the ion gyroradius $(\sim 2 \mathrm{~mm})$, which imposes challenging requirements in the case of SSX plasmas.

We are also planning to carry out merging experiments where two Taylor states will be fired from two opposite ends of the linear SSX device using two magnetized coaxial plasma guns. The main goal of these experiments is to check if the two relaxed states merge together to form one longer object. If the two Taylor states do not merge we expect to observe increased compressive heating, as the plasma will be compressed against another oppositely moving plasma (in place of a metallic wall in the present experiments).

In addition to these projects, our group is performing proton orbit simulations ${ }^{32}$ in the Taylor state fields to understand the particle dynamics in more detail. A typical trajectory of a trapped particle is shown in Fig. 10. Because the magnetic field varies on length scales close to the ion gyroradius, it is not possible to treat the motion in terms of guiding center trajectories. The fraction of an initial particle distribution that is confined in the Taylor state is only of order $10 \%$ less than the corresponding fraction for the axisymmetric spheromak. We are analyzing the characteristics of the particles that remain confined, with the goal of illuminating the requirements for particle confinement in the Taylor state. Currently, we are tracking test particles in a static Taylor state field. Eventually, we plan to track the particle orbits in dynamical Taylor state fields to investigate the evolution of distributions during compression. 


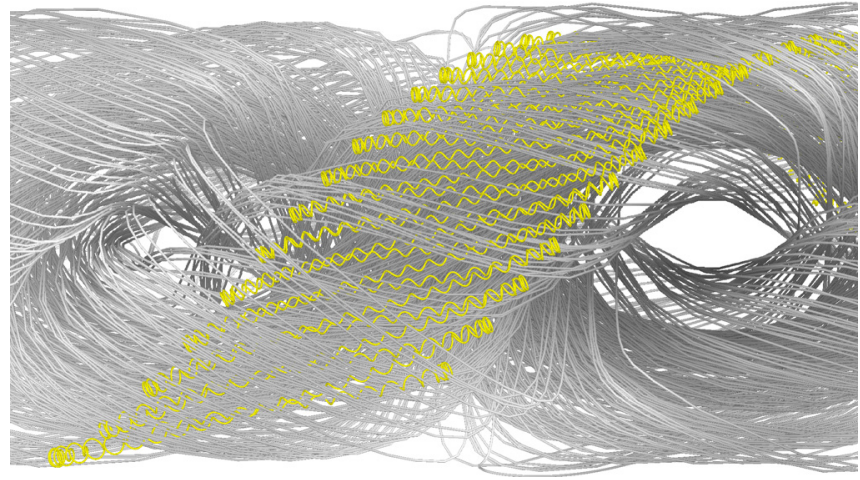

FIG. 10. A typical orbit of a confined proton, with initial parallel and perpendicular velocities of the order of the ion thermal speed, is represented by the yellow trace. The static Taylor state field is shown in grayscale (cropped to show the orbit region), where the lighter values indicate lower field magnitudes. The orbit aligns with the local field over much of the trajectory, but it is clear that the field changes on spatial scales close to the ion gyroscale.

\section{ACKNOWLEDGMENTS}

This work was supported by the Accelerating Low-Cost Plasma Heating and Assembly (ALPHA) Program of the Advanced Research Projects Agency Energy (ARPA-E), Grant No. S00000779. We wish to acknowledge Luke J. Barbano for his support in coding, Emma M. Suen-Lewis for working on the interferometer calibration, Hariharan Srinivasulu and Nicholas Anderson for working on the simulations, Jaron E. Shrock and Katie D. Gelber for working on the apparatus in the SSX lab, David A. Schaffner for several fruitful discussions, and Steven Palmer and Paul Jacobs for their technical support.

\section{REFERENCES}

${ }^{1}$ H.-S. Park, O. A. Hurricane, D. A. Callahan, D. T. Casey, E. L. Dewald, T. R. Dittrich, T. Döppner, D. E. Hinkel, L. F. Berzak Hopkins, S. Le Pape, T. Ma, P. K. Patel, B. A. Remington, H. F. Robey, J. D. Salmonson, and J. L. Kline, Phys. Rev. Lett. 112, 055001 (2014).

${ }^{2}$ O. A. Hurricane, D. A. Callahan, D. T. Casey, P. M. Celliers, C. Cerjan, E. L. Dewald, T. R. Dittrich, T. Döppner, D. E. Hinkel, L. F. B. Hopkins, J. L. Kline, S. L. Pape, T. Ma, A. G. MacPhee, J. L. Milovich, A. Pak, H.-S. Park, P. K. Patel, B. A. Remington, J. D. Salmonson, P. T. Springer, and R. Tommasini, Nature 506, 343 (2014).

${ }^{3}$ J. H. Hammer, C. W. Hartman, J. L. Eddleman, and H. S. McLean, Phys. Rev. Lett. 61, 2843 (1988).

${ }^{4}$ J. H. Hammer, J. L. Eddleman, C. W. Hartman, H. S. McLean, and A. W. Molvik, Phys. Fluids B: Plasma Phys. 3, 2236 (1991).

${ }^{5}$ A. W. Molvik, J. L. Eddleman, J. H. Hammer, C. W. Hartman, and H. S. McLean, Phys. Rev. Lett. 66, 165 (1991).

${ }^{6}$ J. Slough, G. Votroubek, and C. Pihl, Nucl. Fusion 51, 053008 (2011).

${ }^{7}$ G. Votroubek, J. Slough, S. Andreason, and C. Pihl, J. Fusion Energy 27, 123 (2008).
${ }^{8}$ T. J. Awe, R. D. McBride, C. A. Jennings, D. C. Lamppa, M. R. Martin, D. C. Rovang, S. A. Slutz, M. E. Cuneo, A. C. Owen, D. B. Sinars, K. Tomlinson, M. R. Gomez, S. B. Hansen, M. C. Herrmann, J. L. McKenney, C. Nakhleh, G. K. Robertson, G. A. Rochau, M. E. Savage, D. G. Schroen, and W. A. Stygar, Phys. Rev. Lett. 111, 235005 (2013).

${ }^{9}$ M. R. Gomez, S. A. Slutz, A. B. Sefkow, D. B. Sinars, K. D. Hahn, S. B. Hansen, E. C. Harding, P. F. Knapp, P. F. Schmit, C. A. Jennings, T. J. Awe, M. Geissel, D. C. Rovang, G. A. Chandler, G. W. Cooper, M. E. Cuneo, A. J. HarveyThompson, M. C. Herrmann, M. H. Hess, O. Johns, D. C. Lamppa, M. R. Martin, R. D. McBride, K. J. Peterson, J. L. Porter, G. K. Robertson, G. A. Rochau, C. L. Ruiz, M. E. Savage, I. C. Smith, W. A. Stygar, and R. A. Vesey, Phys. Rev. Lett. 113, 155003 (2014).

${ }^{10}$ S. A. Slutz and R. A. Vesey, Phys. Rev. Lett. 108, 025003 (2012).

${ }^{11}$ M. Kaur, L. J. Barbano, E. M. Suen-Lewis, J. E. Shrock, A. D. Light, M. R. Brown, and D. A. Schaffner, Phys. Rev. E 97, 011202 (2018).

${ }^{12}$ M. Kaur, L. J. Barbano, E. M. Suen-Lewis, J. E. Shrock, A. D. Light, D. A. Schaffner, M. B. Brown, S. Woodruff, and T. Meyer, J. Plasma Phys. 84, 905840114 (2018).

${ }^{13}$ J. B. Taylor, Rev. Mod. Phys. 58, 741 (1986).

${ }^{14}$ T. Gray, M. R. Brown, and D. Dandurand, Phys. Rev. Lett. 110, 085002 (2013).

${ }^{15} \mathrm{H}$. Childs, E. Brugger, B. Whitlock, J. Meredith, S. Ahern, D. Pugmire, K. Biagas, M. Miller, C. Harrison, G. H. Weber, H. Krishnan, T. Fogal, A. Sanderson, C. Garth, E. W. Bethel, D. Camp, O. Rübel, M. Durant, J. M. Favre, and P. Navrátil, in High Performance Visualization-Enabling Extreme-Scale Scientific Insight (Lawrence Berkeley National Laboratory, 2012), pp. 357-372, see https://escholarship.org/uc/item/69r5m58v.

${ }^{16}$ G. F. Chew, M. L. Goldberger, and F. E. Low, Proc. R. Soc. London A 236, 112 (1956).

${ }^{17}$ M. R. Brown and D. A. Schaffner, J. Plasma Phys. 81, 345810302 (2015).

${ }^{18}$ C. G. R. Geddes, T. W. Kornack, and M. R. Brown, Phys. Plasmas 5, 1027 (1998).

${ }^{19}$ M. R. Brown and D. A. Schaffner, Plasma Sources Sci. Technol. 23, 063001 (2014).

${ }^{20}$ C. D. Cothran, M. R. Brown, T. Gray, M. J. Schaffer, and G. Marklin, Phys. Rev. Lett. 103, 215002 (2009).

${ }^{21}$ C. D. Cothran, J. Fung, M. R. Brown, and M. J. Schaffer, Rev. Sci. Instrum. 77, 063504 (2006).

${ }^{22}$ V. H. Chaplin, M. R. Brown, D. H. Cohen, T. Gray, and C. D. Cothran, Phys. Plasmas 16, 042505 (2009).

${ }^{23}$ M. Kaur, K. D. Gelber, A. D. Light, and M. R. Brown, Plasma 1, 229 (2018).

${ }^{24}$ D. A. Schaffner, M. R. Brown, and V. S. Lukin, Astrophys. J. 790, 126 (2014).

${ }^{25}$ M. R. Brown, D. A. Schaffner, and P. J. Weck, Phys. Plasmas 22, 055601 (2015).

${ }^{26}$ A. N. Kolmogorov, V. Levin, J. C. R. Hunt, O. M. Phillips, and D. Williams, Proc. R. Soc. London Ser. A 434, 9 (1991).

${ }^{27}$ D. A. Schaffner, A. Wan, and M. R. Brown, Phys. Rev. Lett. 112, 165001 (2014).

${ }^{28}$ G. Fiksel, D. J. Den Hartog, and P. W. Fontana, Rev. Sci. Instrum. 69, 2024 (1998).

${ }^{29}$ S. C. Hsu, T. A. Carter, G. Fiksel, H. Ji, R. M. Kulsrud, and M. Yamada, Phys. Plasmas 8, 1916 (2001).

${ }^{30}$ D. W. Mason, J. Nucl. Energy, Part C 6, 553 (1964).

${ }^{31}$ R. L. Stenzel, R. Williams, R. Agero, K. Kitazaki, A. Ling, T. McDonald, and J. Spitzer, Rev. Sci. Instrum. 53, 1027 (1982).

${ }^{32}$ A. D. Light et al., "Confinement properties and particle orbits in a relaxed Taylor state," Phys Plasmas (to be submitted).

${ }^{33}$ C. Hansen, G. Marklin, B. Victor, C. Akcay, and T. Jarboe, Phys. Plasmas 22, 042505 (2015). 